\title{
Sea Lion Optimization Algorithm
}

\author{
Raja Masadeh $^{1}$ \\ Computer Science Department, The World Islamic Sciences \\ and Education University, Amman, Jordan
}

\author{
Basel A. Mahafzah ${ }^{2}$, Ahmad Sharieh ${ }^{3}$ \\ Computer Science Department \\ The University of Jordan, Amman, Jordan
}

\begin{abstract}
This paper suggests a new nature inspired metaheuristic optimization algorithm which is called Sea Lion Optimization (SLnO) algorithm. The SLnO algorithm imitates the hunting behavior of sea lions in nature. Moreover, it is inspired by sea lions' whiskers that are used in order to detect the prey. SLnO algorithm is tested with 23 well-known test functions (Benchmarks). Optimization results show that the SLnO algorithm is very competitive compared to Particle Swarm Optimization (PSO), Whale Optimization Algorithm (WOA), Grey Wolf Optimization (GWO), Sine Cosine Algorithm (SCA) and Dragonfly Algorithm (DA).
\end{abstract}

Keywords-Optimization; Metaheuristic optimization algorithms; Benchmarks; Sea Lion Optimization Algorithm (SLnO)

\section{INTRODUCTION}

Metaheuristic optimization algorithms are becoming more popular in application because they depend on simple concepts and easy to implement. They do not demand gradient information. They can bypass local optima and they can be applied in a wide range of issues covering various disciplines [1-5].

Metaheuristic optimization algorithms are introduced in order to solve optimization problems by imitating physical or biological phenomena [6-11]. Therefore, these algorithms are categorized into three classes; evolution- based, physicsbased, and swarm-based methods [1, 2, 12-13]. Evolutionbased techniques are inspired by the natural evolution' laws. The search operation begins by randomly generating population that is improved is through subsequent descent. Usually, these techniques are characterized by combining the best individuals to form the next individuals' generation. This leads the population over the generations. The most common algorithms of evolution-inspired are Genetic Algorithms (GA) [14], Evolution Strategy (ES) [15], Genetic Programming (GP) [16], Biogeography-Based Optimizer (BBO) [17] and Probability-Based Incremental Learning (PBIL) [18].

Physics-based methods mimic the physical principles in the world. Some of the most common techniques are Ray Optimization (RO) [19], Black Hole (BH) [20], Small-World Optimization Algorithm (SWOA) [21], Simulated Annealing (SA) [22], Big-Bang Big-Crunch (BBBC) [23], Gravitational Search Algorithm (GSA) [24], Charged System Search (CSS) [25] and Curved Space Optimization (CSO) [26].

Swarm- based methods are the third class of nature inspired techniques which imitate the social behavior of animals in nature. The most common technique is Particle Swarm Optimization (PSO) [27] which is mimics the bird flocking's social behavior. PSO employs number of particles which indicate to the candidate solutions that wing in the search space in order to detect the best solution that represent the optimal solution. Moreover, at the same time, they all track the best solution in their routes. Ant Colony Optimization (ACO) algorithm [28] is considered as another common swarm-based technique. ACO imitates the social behavior of ants in their colony. The most significant characteristic of ants is in finding the nearest route from the colony to the food's source; which is the major inspiration of this technique. New metaheuristic optimization algorithm is proposed by [29]. The proposed algorithm called Vocalization of humpback Whale Optimization Algorithm (VWOA) which mimics the vocalization behavior of humpback whales in nature. VWOA employs number of humpback whales as candidate solutions. Over the course of iterations, the first three solutions estimate the location of the female and update their location depends on the humpback female's position. Then, they force the female to join their pods.

There are other metaheuristic algorithms that are inspired by the behaviors of human. Some of these algorithms are Teaching Learning Based Optimization (TLBO), Interior Search Algorithm (ISA), League Championship Algorithm (LCA), Harmony Search (HS) and Colliding Bodies Optimization (CBO).

Metaheuristic algorithms that based on population share popular features regardless of their nature. The search operation has two main phases; exploration and exploitation [9-10]. The operators should always be part of the optimizer in order to globally explore the search space. In this phase, movements should be randomly chosen. Then the exploitation phase should be applied after the exploration phase, this phase is to investigate the found search space area in details [12]. In other words the Exploitation is applied on the region that is found by the exploration phase. Any metaheuristic algorithm faces a challenge in making balance between exploration and exploitation due to the stochastic nature of the optimization process [13].

This paper introduces a novel metaheuristic optimization algorithm that is called Sea Lion Optimization (SLnO) algorithm imitating the hunting behavior of sea lions. Upon of our knowledge, there is no study on this subject. The strength point of this algorithm is the artificial hunting behavior with random or the best search agent in order to hunt the bait ball (prey) and the usage of the whiskers of sea lions and their vocalizations. The performance of the SLnO algorithm is evaluated in this work by solving 23 well-known optimization problems. The results show that SLnO algorithm is very competitive compared to other popular metaheuristic algorithms. 
The rest of the paper is organized as follows. Section 2 introduced a description of Sea Lion Optimization (SLnO) algorithm in this research. Benchmarks functions and the optimization results are described and discussed in Section 3. Finally, Section 4 draws the conclusion of this work.

\section{Sea Lion Optimization (SLnO) Algorithm}

In this section, the inspiration of the Sea Lion optimization (SLnO) method is first discussed. Then, the mathematical model for SLnO is provided.

\section{A. Inspiration}

Sea lion is considered as one of the most intelligent animals [30]. Sea lions live in huge colonies which have thousands of members [30]. There are plenty of subgroups that have their own hierarchy within them. Sea lions can also navigate around these subgroups several times in their lives. The navigation of sea lions relies on their sex, age and the function that they have for the whole colony $[30,31]$.

The most important characteristic of sea lions is how quickly they respond to fish movements [32]. In other words, they have the ability to locate fish and react immediately, in order to gather them towards shallow water to be near the shore and the surface of ocean. Moreover, they have wonderful senses that help them to find out prey such as fishes even in dark underwater. Their eyes indicate forward the prey; in which they can easily focus on their prey. More precisely, they can open their pupils widely to allow a lot of light into their eyes for a clear underwater vision [33].However, sometimes vision in murky environment is not clear enough $[33,34]$. For this reason, sea lions depend on their super sensitive whiskers which are the most significant characteristic of them [35]. These whiskers help them to feel exactly the positions of prey. When the preys swim around them, they leave wakes or waves behind them. Thus, sea lions can follow them using their whiskers [36].

The longest whiskers of all mammals are $30 \mathrm{~cm}$ [37]. They can move them forwards and backwards. Sea lions can use them to specify the size, shape and position of prey. In addition; cross section of facial whiskers for sea lions is oval; which is different from other mammals that have circular facial whiskers [36, 38]. Researchers have illustrated that this is the best form to detect the speed and direction of waves [36].

The other characteristic of sea lions is their ability to move efficiently and quickly over water [33, 39]. Back flippers are employed for guidance, while front flippers are employed for pushing their selves. They have the ability to chase prey at velocity of around $30 \mathrm{mph}$ and they are flexible enough to alter their directions quickly. For this reason, sea lions employ their whiskers [39].

Hunting together as groups of sea lions increase the opportunities of obtaining more prey especially when there are huge numbers of fishes. Usually, sea lions chasing together by collecting prey in to narrow balls and catching the individuals' prey that located on the edges [33, 40, 41]. Sea lions know when to hunt together and usually do that when prey is plenty. However, they hunt individually when the prey is few.
The main phases of hunting behavior of sea lions are as shown in Fig.1 and as follows:

- Tracking and chasing the prey using their whiskers.

- Calling other members that joined their subgroup, pursing and encircling the prey.

- Attack towards the prey.

In this work this hunting technique of sea lions is mathematically modeled in order to design SLnO algorithm and perform optimization.

\section{B. Mathematical model for SLnO algorithm}

In this subsection the mathematical models for the social hierarchy, tracking, encircling, and attacking prey are provided. Then, the SLnO algorithm is outlined.

1) Detecting and tracking phase: As mentioned above, sea lions are used their whiskers to detect the size, shape and position of prey. As shown in Fig. 2, when the whiskers direction is on the opposite direction of water waves, this helps sea lion to sense the existing prey and to detect their position. However, the whiskers vibrated less than when its orientation on the same current orientation.

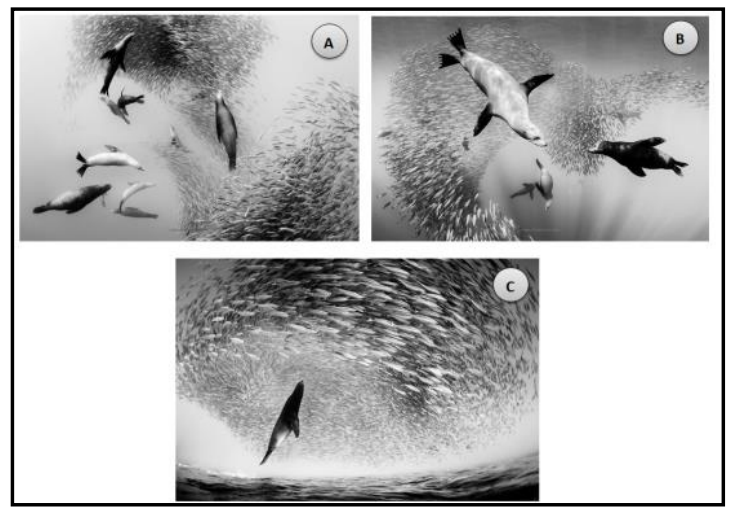

Fig. 1. Hunting behavior of Sea Lions: (A) Chasing, Approaching, and Tracking Prey, (B) Encircling, (C) Stationary Situation and Attack.

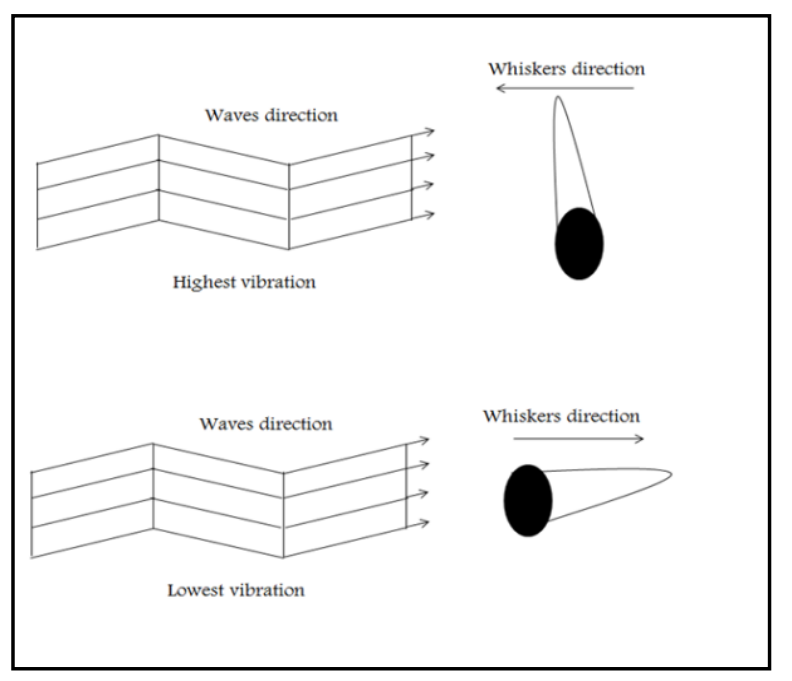

Fig. 2. The Relation between the Whisker' Orientation and the Current' Orientation. 
Sea lion can identify the position of prey and call other members that will join its subgroup to chase and hunt the prey. This sea lion is considered as a leader for this hunting mechanism and other members update their positions towards the target prey. SLnO algorithm assumes the target prey is the current best solution or close to optimal solution. This behavior is represented mathematically using Eq. (1).

$\overrightarrow{D \imath s t}=|\overrightarrow{2 B} \cdot \overrightarrow{P(t)}-\overrightarrow{S L(t)}|$

Where $\overrightarrow{D l s t}$ indicates to the distance between the target prey and the sea lion; $\overrightarrow{P(t)}$ and $\overrightarrow{S L(t)}$ represent the positions vectors of the target prey and sea lion, respectively; the current iteration is denoted as $t$ and $\vec{B}$ is random vector in [0, 1] which is multiplied by 2 to increase the search space that help search agents to find optimal or near optimal solution.

At the next iteration, the sea lion moves toward the target prey to be nearest. This behavior is modeled mathematically as in Eq. (2).

$\overrightarrow{S L(t+1)}=\overrightarrow{P(t)}-\overrightarrow{D \imath s t} \cdot \vec{C}$

Where $(\mathrm{t}+1)$ represents the next iteration and $\vec{C}$ is decreased linearly from 2 to 0 over the course of iterations because this decreasing obliges the sea lion' leader to move towards the current prey and surround them.

2) Vocalization phase: Sea lions are considered amphibians. In other words, Sea lions live in water and on land. Their sounds move four times faster in water than in air [42].Sea lions communicate with each other using various vocalizations especially when they are chasing and hunting as a subgroup [43]. Furthermore, they use their sound to call other members that stay on the shore. For this reason, sea lions chase and confine prey to become close to the surface of ocean. In addition, they have small ears which capable to detect sounds under and above water [30, 33]. Thus, when a sea lion identifies a prey, he calls other members to encircle and attack the prey [30, 44]. This behavior is modeled mathematically as in Eqs. (3), (4) and (5).

$\left.\overrightarrow{S P_{\text {leader }}}=\mid\left(\overrightarrow{V_{1}}\left(1+\overrightarrow{V_{2}}\right)\right) / \overrightarrow{V_{2}}\right) \mid$

$\vec{V}_{1}=\sin \theta$

$\vec{V}_{2}=\sin \emptyset$

Where $\overline{S P_{\text {leader }}}$ indicates to the speed of sound of sea lion leader, $\overrightarrow{V_{1}}$ and $\overrightarrow{V_{2}}$ represents the speed of sounds in water and in air, respectively. More precisely, as shown in Fig. 3, when the sea lion makes a sound, this is reflected to the other medium which is the air (for calling other members that are at the shore) and refracted at the same medium for calling members who are under water. Thus, the first case is represented using $(\sin \emptyset)$; while the other case is represented using $(\sin \theta)$.

3) Attacking phase (Exploitation phase): Sea lions will be able to recognize the position of target prey and encircle them. The hunt method is guided by the leader (best search agent) who detects the prey and tells others members about them.
Usually the target prey is considered the current candidate best solution. However, a new search agent can be defined, detects better preys and encircle them.

In order to mathematically model the hunting behavior of sea lions, two phases are introduced as follows:

a) Dwindling encircling technique: This behavior depends on the value of $\vec{C}$ in Eq. (2). More precisely, $\vec{C}$ is decreased linearly from 2 to 0 over the course of iterations. Thus, this decreasing leads the leader of sea lion to move towards the prey and encircle them. Thus, the incoming location of a sea lion (search agent) can be located anywhere between the premier location of the agent and the location of the present best agent.

b) Circle updating position: As illustrated in Fig. 4, sea lions chase bait ball of fishes and hunt them starting from edges. Eq. (6) is proposed in this regard.

$\overrightarrow{S L}(t+1)=|\vec{P}(t)-\overrightarrow{S L}(t)| \cdot \cos (2 \pi m)+\vec{P}(t)$

Where $|\vec{P}(t)-\overrightarrow{S L}(t)|$ represents the distance between the best optimal solution (target prey) and the search agent (sea lion), | indicates to the absolute value and $m$ is a random number in $[-1,1]$. The sea lion swims around prey (bait ball) along circle shaped path in order to start hunting prey that are at the edge of the bait ball. For this reason, $\cos (2 \pi m)$ is used to represent this behavior mathematically.

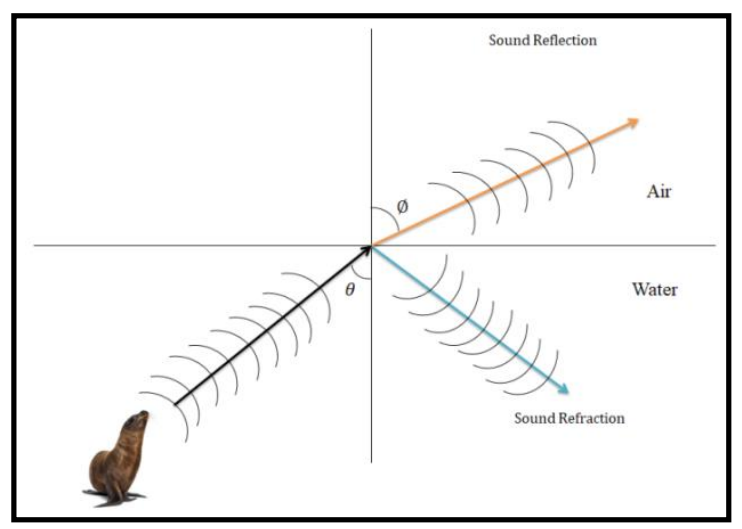

Fig. 3. Sea Lion' Sounds Waves Reflection and Refraction in Two different Medium.

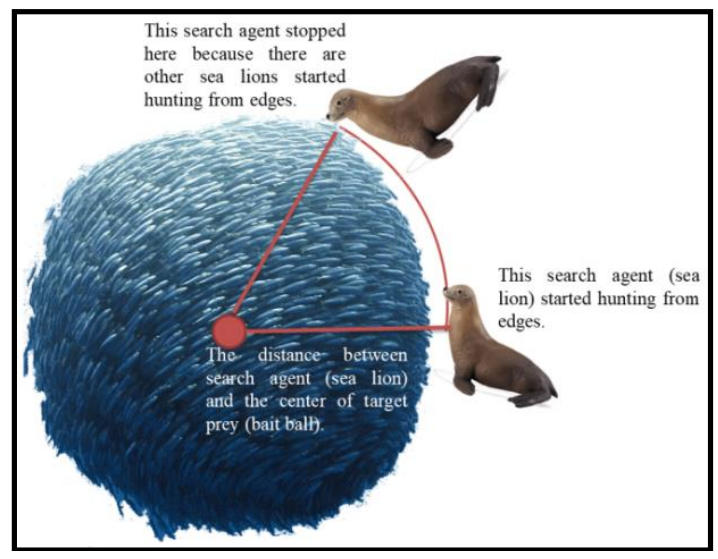

Fig. 4. Circle updating Position of Sea Lions based on Bait Ball (Prey). 
4) Searching for prey (Exploration phase): In nature, sea lions search randomly employing their whiskers and swimming zigzagging to find prey. Thus, in this study, $\vec{C}$ is employed with the random values. In case $\vec{C}$ is greater than one or less than negative one, this leads to force sea lions to move away from the target prey and the sea lion' leader. Therefore, this situation obliges sea lions to search for other prey.

In exploitation phase, the sea lions update their positions based on the best search agent. However, in exploration phase, the search agents update their positions according to a selected randomly sea lion. In other words, when $\vec{C}$ is greater than one, this leads that SLnO algorithm to perform a global search agent and find the global optimal solution. Eq. (7) and Eq. (8) are proposed in this regard.

$\overrightarrow{D l s t}=\left|\overrightarrow{2 B} \cdot \overrightarrow{S L}_{r n d}(t)-\overrightarrow{S L(t)}\right|$

$\overrightarrow{S L}(t+1)=\overrightarrow{S L}_{r n d}(t)-\overrightarrow{D \iota s t} \cdot \vec{C}$

Where $\overrightarrow{S L}_{r n d}(t)$ indicates to random sea lion that is selected from the current population.

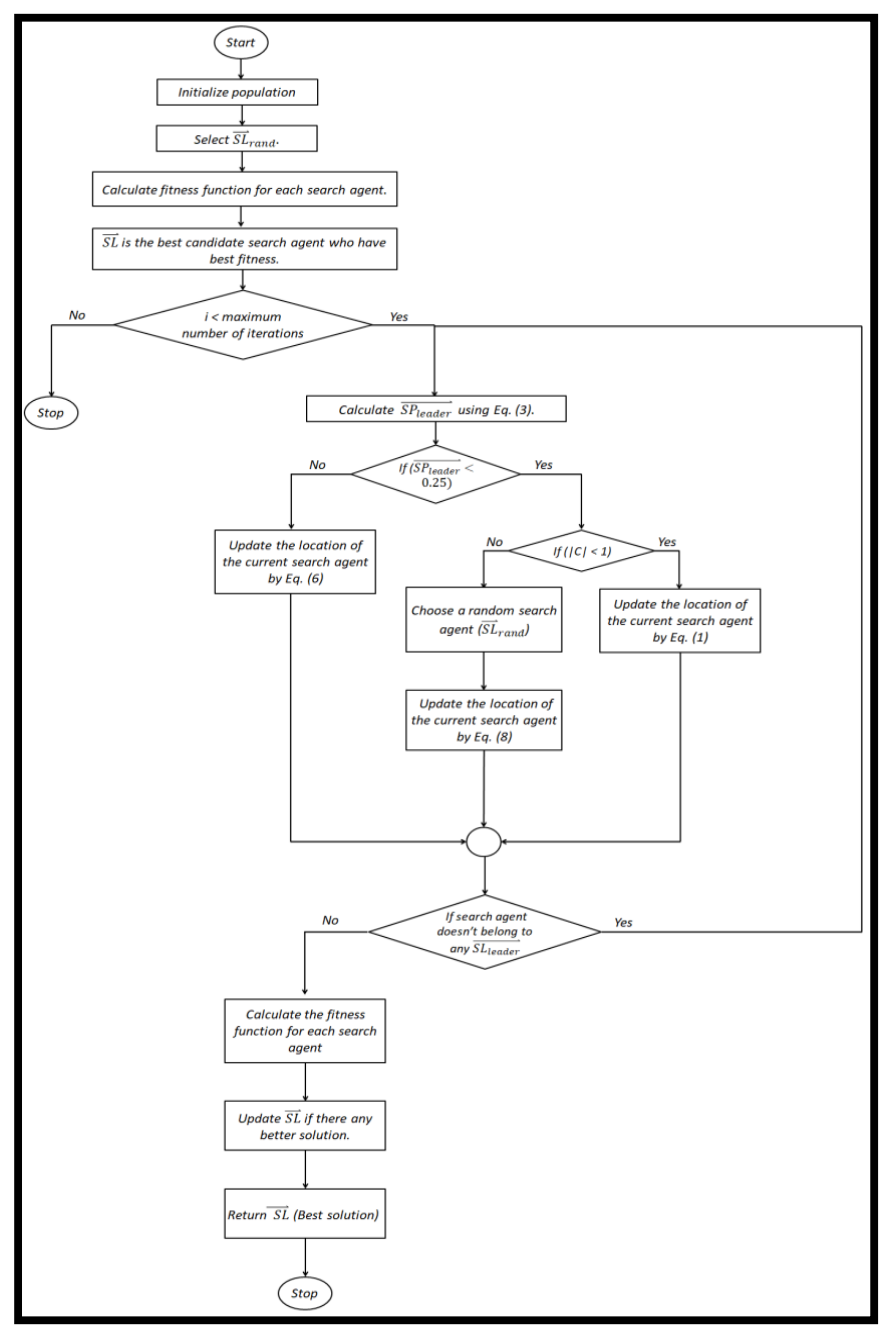

Fig. 5. Flowchart of SLnO Algorithm.
The proposed SLnO algorithm starts with random solutions. Each search agent updates its location based on best solution or random search agent. Parameter $(C)$ is minimized from 2 to 0 over course of iterations to supply both exploration and exploitation phases. More precisely, when the value of $|\vec{C}|$ is greater than one, this means a search agent is chosen randomly. While, when $|\vec{C}|$ is less than one; this means search agents update their locations. Finally, by the satisfaction of an ending criterion, SLnO algorithm is stopped.

Fig. 5 illustrates the flowchart of SLnO algorithm.

\section{EXPERIMENTAL RESULTS}

The proposed SLnO algorithm is benchmarked on 23 benchmark functions that are the classical functions utilized by many researchers $[1,2,45,46]$. SLnO algorithm is compared with recently metaheuristic optimization algorithms; WOA, GWO and PSO. Tables I to III brief the test problems that are denoting the function's cost, range of variation of optimization variables and the optimal value that is denoted as $\mathrm{f}_{\text {min }}$ in previous studies.

In general, these benchmark functions are minimization functions as well as can be categorized into three groups; unimodal, multimodal and fixed-dimension multimodal functions. Fig. 6 to Fig. 8 show the 2D plots of function's cost for 23 benchmark functions which considered in this work.

The experiments are conducted using Matlab R2016a. For all algorithms, the proposed SLnO and existing WOA, GWO and PSO algorithms, a population size is 300 and maximum iteration equal to 500. Each of these algorithms was run 30 times on each benchmark function.

TABLE I. DETAILS OF UNIMODAL BENCHMARK FunCtions (MIRJALILI AND LEWIS, 016)

\begin{tabular}{|c|l|l|l|}
\hline Function & $V_{-}$no & Range & $f_{\min }$ \\
\hline$F_{1}(x)=\sum_{i=1}^{n} x_{i}^{2}$ & 30 & {$[-100,100]$} & 0 \\
\hline$F_{2}(x)=\sum_{i=1}^{n}\left|x_{i}\right|+\prod_{i=1}^{n}\left|x_{i}\right|$ & 30 & {$[-10,10]$} & 0 \\
\hline$F_{3}(x)=\sum_{i=1}^{n}\left(\sum_{j-1}^{i} x_{j}\right)^{2}$ & 30 & {$[-100,100]$} & 0 \\
\hline$F_{4}(x)=\max _{i}\left\{\left|x_{i}\right|, 1 \leq i \leq n\right\}$ & 30 & {$[-100,100]$} & 0 \\
\hline$F_{5}(x)=\sum_{i=1}^{n-1}\left[100\left(x_{i+1}-x_{i}^{2}\right)^{2}\right.$ & 30 & {$[-30,30]$} & 0 \\
\hline$F_{7}(x)=\sum_{i=1}^{n} i x_{i}^{4}+$ random $(0,1)$ & 30 & {$[-1.28$,} & 0 \\
\hline$F_{6}(x)=\sum_{i=1}^{n}\left(\left[x_{i}+0.5\right]\right)^{2}$ & 30 & {$[-100,100]$} & 0 \\
\hline
\end{tabular}


TABLE II. DETAILs OF Multimodal BenChMARK FunCtions (MIRJALILI AND LEWIS, 016)

\begin{tabular}{|c|c|c|c|}
\hline Function & $V_{-} n o$ & Range & $f_{\min }$ \\
\hline$F_{8}(x)=\sum_{i=1}^{n}-x_{i} \sin \left(\sqrt{\left|x_{i}\right|}\right.$ & 30 & {$[-500,500]$} & - \\
\hline $\begin{array}{c}F_{9}(x)=\sum_{i=1}^{n}\left[x_{i}^{2}-10 \cos \left(2 \pi x_{i)}\right.\right. \\
+10]\end{array}$ & 30 & {$[-5.12,5.12]$} & 0 \\
\hline $\begin{array}{l}F_{10}(x) \\
=-20 \exp \left(-0.2 \sqrt{\left.\frac{1}{n} \sum_{i=1}^{n} x_{i}^{2}\right)}\right. \\
-\exp \left(\frac{1}{n} \sum_{i=1}^{n} \cos \left(2 \pi x_{i}\right)\right)+20 \\
+e\end{array}$ & 30 & {$[-32,32]$} & 0 \\
\hline $\begin{array}{l}F_{11}(x) \\
=\frac{1}{4000} \sum_{i=1}^{n} x_{i}^{2} \\
-\prod_{i=1}^{n} \cos \left(\frac{x_{i}}{\sqrt{i}}\right)+1\end{array}$ & 30 & {$[-600,600]$} & 0 \\
\hline $\begin{array}{l}F_{12}(x)=\frac{x}{n}\left(\left\{10 \sin \left(\pi y_{i}\right)+\right.\right. \\
\sum_{i=1}^{n}\left(y_{i}-1\right)^{2}[1+ \\
\left.\left.\left.10 \sin ^{2}\right]\left(\pi y_{i+1}\right)\right]+\left(y_{n}-1\right)^{2}\right\}+ \\
\left.\sum_{i=1}^{n} u\left(x_{i}, 10,100,4\right)\right\} \\
\quad y_{i}=1+\frac{x_{i}+1}{4} u\left(x_{i}, a, k, m\right) \\
\quad=\left\{\begin{array}{c}k\left(x_{i}-a\right)^{m} x_{i}>a \\
0-a<x_{i}<a \\
k\left(-x_{i}-a\right)^{m} x_{i}<-a\end{array}\right.\end{array}$ & 30 & {$[-50,50]$} & 0 \\
\hline $\begin{array}{l}F_{13}(x) \\
=0.1\left\{\sin ^{2}\left(3 \pi x_{1}\right)\right. \\
+\sum_{i=1}^{n}\left(x_{i}-1\right)^{2}[1 \\
\left.+\sin ^{2}\left(3 \pi x_{i}+1\right)\right] \\
\left.+\left(x_{n}-1\right)^{2}\left[1+\sin ^{2}\left(2 \pi x_{n}\right)\right]\right\} \\
+\sum_{i=1}^{n} u\left(x_{i}, 5,100,4\right)\end{array}$ & 30 & {$[-50,50]$} & 0 \\
\hline
\end{tabular}

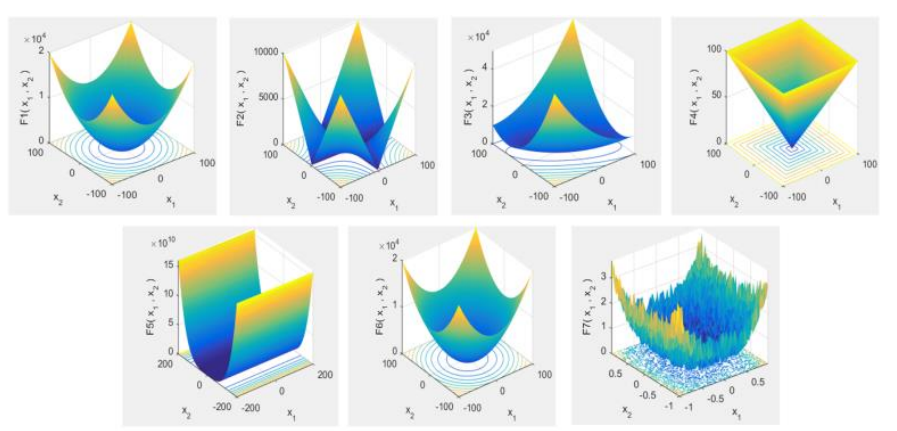

Fig. 6. 2D Representations of Benchmark Mathematical unimodal Functions.
TABLE III. DETAILS OF FIXED-DIMENSION MULTIMODAL BENCHMARK FUNCTIONS (MIRJALILI AND LEWIS, 016)

\begin{tabular}{|c|c|c|c|}
\hline Function & $V_{-} n \boldsymbol{o}$ & Range & $f_{\min }$ \\
\hline $\begin{array}{l}F_{14}(x) \\
=\left(\frac{1}{500}\right. \\
\left.+\sum_{j=1}^{25} \frac{1}{j+\sum_{i=1}^{2}\left(x_{i}-a_{i j}\right)^{6}}\right)^{-1}\end{array}$ & 2 & {$[-65,65]$} & 1 \\
\hline $\begin{array}{l}F_{15}(x) \\
=\sum_{i=1}^{11}\left[a_{i-} \frac{x_{1}\left(b_{i}^{2}+b_{i} x_{2}\right)}{b_{i}^{2}+b_{i} x_{3}+x_{4}}\right]^{2}\end{array}$ & 4 & {$[-5,5]$} & 0.00030 \\
\hline $\begin{array}{r}F_{16}(x)=4 x_{1}^{2}-2.1 x_{1}^{4}+\frac{1}{3} x_{1}^{6}+x_{1} x_{2} \\
-4 x_{2}^{2}+4 x_{2}^{4}\end{array}$ & 2 & {$[-5,5]$} & -1.0316 \\
\hline $\begin{aligned} F_{17}(x)=\left(x_{2}-\frac{5.1}{4 \pi^{2}}\right. & \left.x_{1}^{2}+\frac{5}{\pi} x_{1}-6\right)^{2} \\
& +10(1 \\
& \left.-\frac{1}{8 \pi}\right) \cos x_{1} \\
& +10\end{aligned}$ & 2 & {$[-5,5]$} & 0.398 \\
\hline $\begin{aligned} F_{18}(x)=\left[1+\left(x_{1}+\right.\right. & \left.x_{2}+1\right)^{2}(19 \\
& -14 x_{1}+3 x_{1}^{2} \\
& -14 x_{2} \\
& +6 x_{1} x_{2} \\
& \left.\left.+3 x_{2}^{2}\right)\right]\end{aligned}$ & 2 & {$[-2,2]$} & 3 \\
\hline $\begin{array}{l}F_{19}(x) \\
=-\sum_{i=1}^{4} c_{i} \exp \left(-\sum_{j=1}^{3} a_{i j}\left(x_{j}\right.\right. \\
\left.\left.-p_{i j}\right)^{2}\right)\end{array}$ & 3 & {$[1,3]$} & -3.86 \\
\hline $\begin{array}{l}F_{20}(x) \\
=-\sum_{i=1}^{4} c_{i} \exp \left(-\sum_{j=1}^{6} a_{i j}\left(x_{j}\right.\right. \\
\left.\left.-p_{i j}\right)^{2}\right)\end{array}$ & 6 & {$[0,1]$} & -3.32 \\
\hline $\begin{array}{c}F_{21}(x)=-\sum_{i=1}^{5}\left[\left(X-a_{i}\right)\left(X-a_{i}\right)^{T}\right. \\
\left.+c_{i}\right]^{-1}\end{array}$ & 4 & {$[0,10]$} & -10.4028 \\
\hline $\begin{array}{c}F_{22}(x)=-\sum_{i=1}^{7}\left[\left(X-a_{i}\right)\left(X-a_{i}\right)^{T}\right. \\
\left.+c_{i}\right]^{-1}\end{array}$ & 4 & {$[0,10]$} & -10.5363 \\
\hline $\begin{array}{c}F_{23}(x)=-\sum_{i=1}^{10}\left[\left(X-a_{i}\right)\left(X-a_{i}\right)^{T}\right. \\
\left.+c_{i}\right]^{-1}\end{array}$ & 4 & {$[0,10]$} & -10.1532 \\
\hline
\end{tabular}

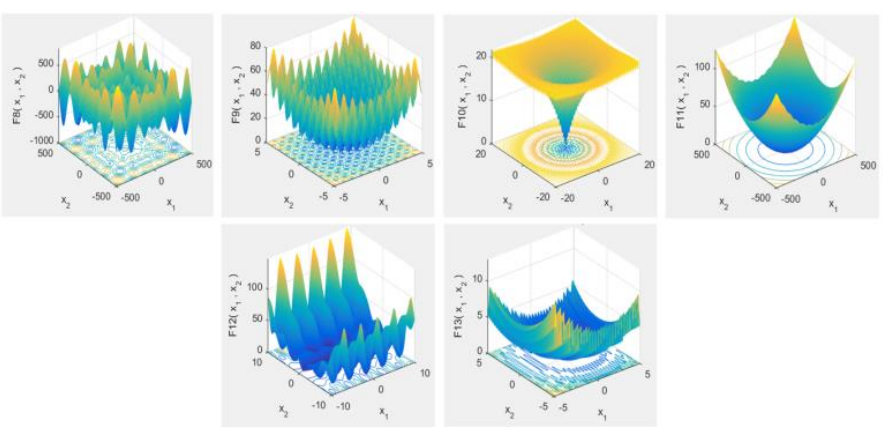

Fig. 7. 2D Representations of Benchmark Mathematical Multimodal Functions. 


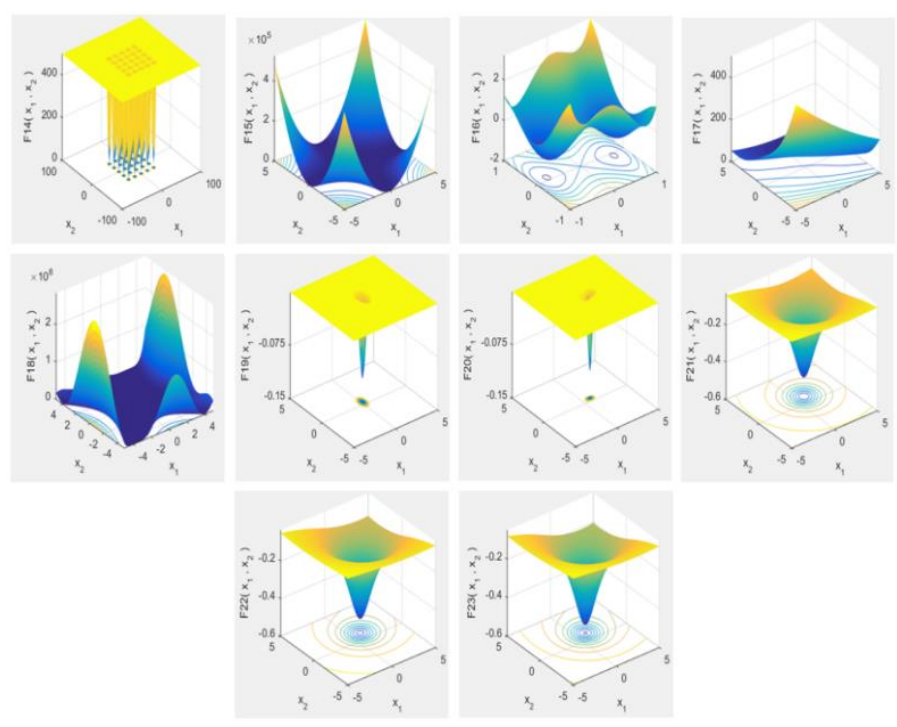

Fig. 8. 2D Representations of Benchmark Mathematical Fixed-Dimension Multimodal Functions.

\section{A. Evaluation of Exploitation Capability (Functions F1-F7)}

Functions F1-F7 are unimodal functions in which they have only one global optimum. Moreover, they allow evaluating the capability of exploitation of inspected metaheuristic optimization algorithms. According to the results of Table IV, SLnO is able to offer competitive outcomes. SLnO was the most efficient optimizer compared to the well-known optimizers especially functions F1, F2, F4 and F5 as well as at least it was the second best optimizer in most benchmark functions.

\section{B. Evaluation of Exploitation Capability (Functions F8-F23)}

In contrast to the unimodal functions, multimodal functions involve many local optima with increasing the number exponentially with the size of problem. Thus, this type of benchmark functions turn very suitable and useful in case the target is to evaluate the exploration ability of an optimization algorithm. According to the outcomes in Table $\mathrm{V}$, for functions F8-F23, the SLnO algorithm has a good exploration capability. As seen in Table V, it is obvious that SLnO is the most efficient or the second best optimizer in the majority of benchmark functions.

\section{Convergence Behavior Analysis}

In this subsection the convergence behavior of SLnO algorithm is investigated. Based on Fig. 9, it is observed that search agent of the SLnO algorithm tends to search favorable regions of design space, as well as utilizes the best one. In the early stages of the optimization operation, these search agents change suddenly and afterward progressively converge. Based on [47], this behavior can ensure that a SLnO algorithm which is based on the population converges to a point in search space. In Fig. 9, convergence curves of the proposed algorithms, PSO, WOA, SCA, DA and GWO algorithms are compared for 23 benchmarks problem. It is obvious that SLnO algorithm is enough competitive with the existing metaheuristic optimization algorithms.
The convergence curves of SLnO, SCA, DA, WOA, GWO and PSO algorithms are presented in Fig.9, in order to show these algorithms' convergence rate. Knowing that the "average best-so-far" denotes the best solutions' average that acquired at each iteration over 30 runs. As shown in these figures, when optimizing the test benchmarks functions, SLnO algorithm illustrates two convergence behaviors. In the first behavior, the SLnO algorithm's convergence tends to be instant as iteration increases as observed in F3, F4, F14, F21, F22 and F23. This is probably due to the adaptation technique that suggested for SLnO algorithm. At the initial stage of each iteration, the adaptation technique helps to search for optimizing regions of search space, then after passing almost half or slightly less of the iterations it convergence towards the optimal solution. In the second behavior, the convergence tends towards optimal solution rapidly from the initial stages of iterations. This behavior is evident in the rest benchmark functions.

As an outline, the outcomes of this subsection discovered various characteristics of the suggested SLnO algorithm. The exploration of SLnO algorithm is high because the location updating technique of sea lions using Eq. (8). This formula requires sea lions to proceed randomly around each other through the initial stages of iterations. However, high exploitation and convergence are intensified in the reminder of iterations using Eq. (6). This leads the sea lions to quickly relocation themselves around bait ball in circular shaped path in order towards the best solution. The SLnO algorithm illustrates avoidance of high local optimal solution and speed of convergence simultaneously over the course of iterations.

The outcomes prove the performance of the SLnO algorithm in solving several test functions compared to PSO, WOA and GWO algorithms. PSO algorithm doesn't have operators to dedicate particular iterations to exploitation or exploration. More precisely, PSO employs one equation to update the search agents' locations, which leads to increase the stagnation in local optima. While, WOA and GWO algorithms have good results due to they have operators to consecrate particular iterations to exploitation or exploration. However, the SLnO has better results than WOA and GWO in the most benchmark functions because it has fewer operators that assist to both exploitation and exploration.

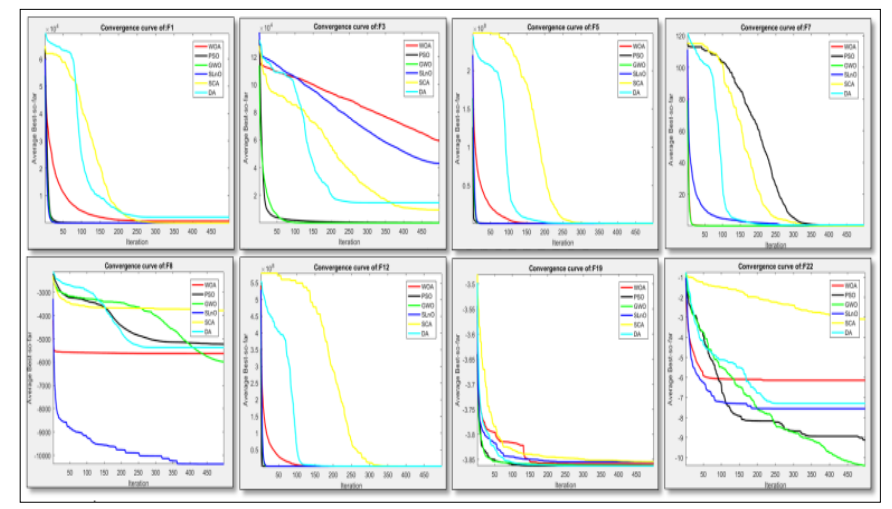

Fig. 9. Comparison of Convergence Curves of SLnO Algorithms and Recently Algorithms Obtained in Some of the Benchmark Problems. 
TABLE IV. COMPARISON OF OPTIMIZERS' RESULTS OBTAINED FOR UNIMODAL BENCHMARK FUNCTIONS

\begin{tabular}{|l|l|l|l|l|l|l|l|l|l|l|l|l|}
\hline Fs & \multicolumn{2}{l}{ SLnO } & \multicolumn{2}{l}{ SCA } & \multicolumn{2}{l|}{ PSO } & \multicolumn{2}{l|}{ WOA } & \multicolumn{2}{l|}{ DA } \\
\hline & Avg. & Std. & Avg. & Std. & Avg. & Std. & Avg. & Std. & Avg. & Std. & Avg. & Std. \\
\hline F1 & $\mathbf{2 . 1 8 E - 4 5}$ & $\mathbf{5 . 7 5 E - 4 5}$ & $9.832 \mathrm{E}-04$ & $13.254 \mathrm{E}-04$ & $\begin{array}{l}2.23 \mathrm{E}- \\
09\end{array}$ & $\begin{array}{l}4.32 \mathrm{E}- \\
09\end{array}$ & $2.04 \mathrm{E}-23$ & $6.39 \mathrm{E}-23$ & $\begin{array}{l}7.961 \mathrm{E}- \\
05\end{array}$ & $11.36 \mathrm{E}-05$ & $2.20 \mathrm{E}-28$ & $4.12 \mathrm{E}-28$ \\
\hline F2 & $\mathbf{1 . 4 5 E - 3 7}$ & $\mathbf{3 . 9 8 E - 3 7}$ & $17.359 \mathrm{E}-04$ & $17.984 \mathrm{E}-04$ & $\begin{array}{l}5.65 \mathrm{E}- \\
05\end{array}$ & $\begin{array}{l}5.82 \mathrm{E}- \\
05\end{array}$ & $9.93 \mathrm{E}-37$ & $5.98 \mathrm{E}-35$ & $\begin{array}{l}14.37 \mathrm{E}- \\
04\end{array}$ & $15.37 \mathrm{E}-04$ & $9.04 \mathrm{E}-36$ & $6.94 \mathrm{E}-29$ \\
\hline F3 & $-2.51 \mathrm{E}-04$ & $1.67 \mathrm{E}-04$ & 11.2354 & 13.5478 & $\begin{array}{l}8.73309 \\
3.78789\end{array}$ & $\mathbf{- 2 . 7 1 E - 0 4}$ & $\mathbf{2 . 7 7 E - 0 4}$ & 12.9876 & 15.0128 & $-6.04 \mathrm{E}-04$ & $2.01 \mathrm{E}-04$ \\
\hline F4 & $\mathbf{0 . 0 6 9 3 2 1}$ & $\mathbf{0 . 4 1 9 8 2}$ & 2.3742 & 3.6874 & $\begin{array}{l}1.03091 \\
5\end{array}$ & 0.42617 & 0.35008 & 0.19677 & 1.9876 & 2.2345 & 0.98630 & 0.86921 \\
\hline F5 & $\mathbf{2 6 . 0 6 1 4 6}$ & $\mathbf{0 . 3 1 0 2 4}$ & 66.9875 & 61.5879 & $\begin{array}{l}49.6269 \\
5\end{array}$ & $\begin{array}{l}41.3711 \\
6\end{array}$ & 32.00966 & 3.24879 & 65.2495 & 58.6547 & 33.92145 & 5.00098 \\
\hline F6 & 0.00063 & 0.00030 & 0.99874 & 1.9821 & $\begin{array}{l}\mathbf{3 . 8 2 E}- \\
\mathbf{0 9}\end{array}$ & $\begin{array}{l}\mathbf{6 . 1 7 E}- \\
\mathbf{0 9}\end{array}$ & 0.003638 & 0.00140 & 1.3257 & 2.0275 & 0.98979 & 1.02147 \\
\hline F7 & 0.00045 & 0.00047 & 0.00795 & 0.00925 & 0.04727 & 0.01569 & 0.023175 & 0.00847 & 2.9024 & 2.9999 & $\mathbf{0 . 0 0 0 0 9}$ & $\mathbf{0 . 0 0 0 7 8}$ \\
\hline
\end{tabular}

TABLE V. COMPARISON OF OPTIMIZERS’ RESUlts OBTAINED FOR MULTIMODAl BENCHMARK FunCTIONS

\begin{tabular}{|c|c|c|c|c|c|c|c|c|c|c|c|c|}
\hline \multirow[t]{2}{*}{ Fs } & \multicolumn{2}{|l|}{ SInO } & \multicolumn{2}{|l|}{ SCA } & \multicolumn{2}{|l|}{ PSO } & \multicolumn{2}{|l|}{ WOA } & \multicolumn{2}{|l|}{ DA } & \multicolumn{2}{|l|}{ GWO } \\
\hline & Avg. & Std. & Avg. & Std. & Avg. & Std. & Avg. & Std. & Avg. & Std. & Avg. & Std. \\
\hline F8 & -12389.05 & 382.570263 & 29.3456 & 321.821 & -6981.15 & 848.8447 & -14219.09 & 472.66037 & 37.9524 & 371.9542 & 25.23801 & 295.86792 \\
\hline F9 & $3.78 E-15$ & $1.44 \mathrm{E}-14$ & 46.0247 & 24.2408 & 32.30133 & 8.73574 & $2.66 \mathrm{E}-15$ & $2.43 \mathrm{E}-14$ & 42.3214 & 12.3578 & 4.12021 & 1.90716 \\
\hline F10 & $4.32 \mathrm{E}-15$ & $2.37 \mathrm{E}-15$ & $5.012 \mathrm{E}-05$ & $\begin{array}{l}3.024 \mathrm{E}- \\
05\end{array}$ & $2.85 \mathrm{E}-05$ & $1.81 \mathrm{E}-05$ & $5.00 \mathrm{E}-15$ & $2.94 \mathrm{E}-15$ & 4.89E-05 & $2.02 \mathrm{E}-05$ & $6.42 \mathrm{E}-17$ & $6.31 \mathrm{E}-15$ \\
\hline F11 & 0.00285 & 0.00761 & 1.9574 & 2.3541 & 0.00992 & 0.01116 & 0.00496 & 0.00876 & 1.2783 & 1.9821 & 0.07942 & 1.99659 \\
\hline F12 & -1.00040 & 0.00190 & 1.9872 & 2.3457 & $3.82 \mathrm{E}-11$ & $3.87 \mathrm{E}-11$ & 0.00066 & 0.00299 & 1.2702 & 1.6247 & 0.98764 & 0.10079 \\
\hline F13 & 0.00037 & 0.00046 & 2.1348 & 2.7321 & 0.00366 & 0.02005 & 0.00287 & 0.00396 & 1.3472 & 1.4215 & 0.98937 & 0.42215 \\
\hline F14 & 1.00641 & 0.13622 & 1.0227 & 0.9867 & 1.03113 & 0.18147 & 1.02163 & 0.14584 & 1.0367 & 12.318 & 1.06157 & 15.13597 \\
\hline F15 & 0.00052 & 0.00023 & 0.3156 & 0.8179 & 0.00056 & 0.00024 & 0.00061 & 0.00044 & 0.9043 & 1.3782 & 0.28946 & 0.71108 \\
\hline F16 & -1.0316 & $6.77 \mathrm{E}-16$ & 1.5462 & 7.3589 & -2.0218 & $\begin{array}{l}7.78 \mathrm{E}- \\
16\end{array}$ & 0.04326 & $7.00 \mathrm{E}-16$ & 2.8278 & 2.9817 & 0.92497 & 0.09844 \\
\hline F17 & 0.39686 & $1.69 \mathrm{E}-16$ & 1.8234 & 7.3215 & 0.39799 & $1.79 \mathrm{E}-16$ & 1.438789 & $2.67 \mathrm{E}-16$ & 1.9254 & 8.3897 & 2.73245 & 5.48978 \\
\hline F18 & 3 & 0 & 3.0982 & 1.8245 & 3 & $\begin{array}{l}1.33 \mathrm{E}- \\
15 \\
\end{array}$ & 3 & $4.22 \mathrm{E}-05$ & 3.0261 & 0.1124 & 1.62785 & 9.38998 \\
\hline F19 & -3.8984 & 0.00141 & 1.2761 & 4.3257 & -3.8628 & $3.16 \mathrm{E}-15$ & -1.98761 & 1.00253 & 0.8976 & 0.9951 & 0.20211 & 0.42842 \\
\hline F20 & -3.2599 & 0.06460 & 1.4801 & 0.9207 & -3.25858 & 0.06033 & -2.12657 & 0.06460 & 0.7608 & 0.6247 & 0.85685 & 0.21593 \\
\hline F21 & -10.15317 & 9.52E-05 & 9.3801 & 4.3852 & -8.97126 & 2.17908 & -9.16430 & $9.98 \mathrm{E}-05$ & 7.7785 & 6.8927 & 6.16721 & 2.51935 \\
\hline F22 & -10.27894 & 0.97040 & 6.3875 & 3.3861 & $\begin{array}{l}- \\
10.22709\end{array}$ & 0.96291 & -10.22664 & 0.98040 & 5.3692 & 2.3578 & 4.20056 & 1.24785 \\
\hline F23 & -827.5677 & 1.61753 & 5.9632 & 0.2145 & -9.63822 & 2.04274 & -637.668 & 1.61753 & 3.36980 & 0.8732 & 4.33169 & 0.96579 \\
\hline
\end{tabular}

\section{CONCLUSION}

This research presented a novel swarm based optimization algorithm which mimics the sea lions' hunting behavior. The suggested technique is called Sea Lion Optimization (SLnO) algorithm involved three main factors to simulate the exploration of bait ball using the whiskers of sea lions, encircling bait ball and the vocalization of sea lions. Moreover, this work was conducted on 23 mathematical optimization problems in order to analyze the exploration phase, exploitation phase and the suggested method's convergence behavior. Optimization results showed that SLnO algorithm is competitive comparing with other recently metaheuristic algorithms. 


\section{REFERENCES}

[1] Mirjalili, S., \& Lewis, A. (2016). The whale optimization algorithm. Advances in engineering software, 95, 51-67.

[2] Mirjalili, S., Mirjalili, S. M., \& Lewis, A. (2014). Grey wolf optimizer. Advances in engineering software, 69, 46-61.

[3] Masadeh, R., Sharieh, A., \& Sliet, A. (2017). Grey wolf optimization applied to the maximum flow problem. International Journal of Advanced and Applied Sciences, 4(7), 95-100.

[4] Masadeh, R., Alzaqebah, A., \& Sharieh, A. (2018). Whale Optimization Algorithm for Solving the Maximum Flow Problem. Journal of Theoretical \& Applied Information Technology, 96(8).

[5] Alzaqebah, A., Masadeh, R., \& Hudaib, A. (2018, April). Whale optimization algorithm for requirements prioritization. In 2018 9th International Conference on Information and Communication Systems (ICICS) (pp. 84-89). IEEE.

[6] Aryaf Al-Adwan, Ahmad Sharieh, and Basel A. Mahafzah "Parallel heuristic local search algorithm on OTIS hyper hexa-cell and OTIS mesh of trees optoelectronic architectures" Applied Intelligence, Vol. 49(2), pp. 661-688, 2019.

[7] Aryaf Al-Adwan, Basel A. Mahafzah, and Ahmad Sharieh "Solving traveling salesman problem using parallel repetitive nearest neighbor algorithm on OTIS-Hypercube and OTIS-Mesh optoelectronic architectures" Journal of Supercomputing, Vol. 74(1), pp. 1-36, 2018.

[8] Basel A. Mahafzah, "Performance evaluation of parallel multithreaded A* heuristic search algorithm" Journal of Information Science, SAGE, United Kingdom, Vol. 40(3), pp. 363-375, 2014.

[9] Mohammad A. Alshraideh, Basel A. Mahafzah, Hamzeh S. Eyal Salman, Imad Salah, "Using genetic algorithm as test data generator for stored PL/SQL program units" Journal of Software Engineering and Applications, Vol. 6(2), pp. 65-73, 2013.

[10] Mohammad Alshraideh, Basel A. Mahafzah, and Saleh Al-Sharaeh, "A multiple-population genetic algorithm for branch coverage test data generation" Software Quality Journal, Vol. 19(3), pp. 489-513, 2011.

[11] Basel A. Mahafzah, "Parallel multithreaded IDA* heuristic search: Algorithm design and performance evaluation" International Journal of Parallel, Emergent and Distributed Systems, Vol. 26(1), pp. 61-82, 2011.

[12] Masadeh, R., Hudaib, A., \& Alzaqebah, A. (2018). WGW: A hybrid approach based on whale and grey wolf optimization algorithms for requirements prioritization. Advances in Systems Science and Applications, 18(2), 63-83.

[13] Masadeh, R., Alzaqebah, A., Hudaib, A., \& Rahman, A. A. (2018). Grey Wolf Algorithm for Requirements Prioritization. Modern Applied Science, 12(2), 54.

[14] Holland JH . Genetic algorithms. Sci Am 1992; 267:66-72.

[15] Rechenberg I . Evolutions strategien (1978). Springer Berlin Heidelberg; 1978 p. 83-114.

[16] J.R. Koza, "Genetic programming,"1992.

[17] Simon D . Biogeography-based optimization. IEEE Trans Evol Comput 2008; 12:702-13.

[18] Dasgupta D, Zbigniew M, editors (2013). Evolutionary algorithms in engineering applications. Springer Science \& Business Media.

[19] Kaveh, A., \& Khayatazad, M. (2012). A new meta-heuristic method: ray optimization. Computers \& structures, 112, 283-294.

[20] Hatamlou, A. (2013). Black hole: A new heuristic optimization approach for data clustering. Information sciences, 222, 175-184.

[21] Jiao, L., Wang, L., Gao, X., Liu, J., \& Wu, F. (Eds.). (2006). Advances in Natural Computation: Second International Conference, ICNC 2006, Xi'an, China, September 24-28,2006, Proceedings (Vol. 4222). Springer.

[22] Černý, V. (1985). Thermodynamical approach to the traveling salesman problem: An efficient simulation algorithm. Journal of optimization theory and applications, 45(1), 41-51.

[23] Erol, O. K., \& Eksin, I. (2006). A new optimization method: big bangbig crunch. Advances in Engineering Software, 37(2), 106-111.
[24] Rashedi, E., Nezamabadi-Pour, H., \& Saryazdi, S. (2009). GSA: a gravitational search algorithm. Information sciences, 179(13),2232-2248.

[25] Kaveh, A., \& Talatahari, S. (2010). A novel heuristic optimization method: charged system search. Acta Mechanica, 213(3-4), 267-289.

[26] Moghaddam, F. F., Moghaddam, R. F., \& Cheriet, M. (2012). Curved space optimization: a random search based on general relativity theory. arXiv preprint arXiv:1208.2214.

[27] Kennedy J , Eberhart R . Particle swarm optimization. In: Proceedings of the 1995 IEEE international conference on neural networks; 1995. p. 1942-8.

[28] Dorigo M , Birattari M , Stutzle T . Ant colony optimization. IEEE Comput Intell 2006;1:28-39.

[29] Masadeh, R., Sharieh, A., \& Mahafzah, B. (2019), Humpback Whale Optimization Algorithm Based on Vocal Behavior for Task Scheduling in Cloud Computing. International Journal of Advanced Science and Technology, 13 (3), 121-140.

[30] Pitcher, B. J., Harcourt, R. G., \& Charrier, I. (2012). Individual identity encoding and environmental constraints in vocal recognition of pups by Australian sea lion mothers. Animal Behaviour, 83(3), 681-690.

[31] Folkens, P. A., \& Reeves, R. R. (2002). Guide to marine mammals of the world (No. Sirsi) i9780375411410). National Audubon Society.

[32] Pitcher, T. J., Kalikoski, D., Short, K., Varkey, D., \& Pramod, G. (2009). An evaluation of progress in implementing ecosystem-based management of fisheries in 33 countries. Marine Policy, 33(2), 223-232.

[33] Schusterman, R. J. (1981). Behavioral capabilities of seals and sea lions: a review of their hearing, visual, learning and diving skills. The psychological record, 31(2), 125-143.

[34] Poulter, T. C. (1963). Sonar signals of the sea lion. Science, 139(3556), 753-755.

[35] Marine and Coastal Ecology Research Center, http://www.marineeco.org/, last accessed 15 March 2019.

[36] Lowther, A. D., Harcourt, R. G., Hamer, D. J., \& Goldsworthy, S. D. (2011). Creatures of habit: foraging habitat fidelity of adult female Australian sea lions. Marine Ecology Progress Series, 443, 249-263.

[37] Marine Mammal Research Unit, http://mmru.ubc.ca/, last accessed 15 March 2019.

[38] Sea Lion World, https://www.sealion-world.com/sea-lion-socialstructure/, last accessed 15 March 2019

[39] Biographic, https://www.biographic.com/search/sea\%20lion, last accessed 15 March 2019.

[40] NOAA Fishers, https://www.westcoast.fisheries.noaa.gov/protected spe cies/marine_mammals/pinnipeds/california_sea_lions.html, last accesses 15 March 2019.

[41] NOAA National Ocean Service, https://search.noaa.gov/search?dc=119 $9 \& u t f 8=\% \mathrm{E} 2 \% 9 \mathrm{C} \% 93 \&$ affiliate=oceanservice.noaa.gov\&query $=$ sea + li on, last accessed 15 March 2019.

[42] Rogers, P. H., \& Cox, M. (1988). Underwater sound as a biological stimulus, In Sensory biology of aquatic animals, Springer, New York, NY, 131-149.

[43] Evans, W. E., \& Haugen, R. M. (1963). An experimental study of the echolocation ability of a California sea lion, Zalophus californianus (Lesson). Bulletin of the Southern California Academy of Sciences, 62(4), 165-175.

[44] Pacific Marine Mammal Center, https://www.pacificmmc.org/, last accessed 15 March 2019.

[45] Yao, X., Liu, Y., \& Lin, G. (1999). Evolutionary programming made faster, IEEE Transactions on Evolutionary computation, 3(2), 82-102.

[46] Digalakis, J. G., \& Margaritis, K. G. (2001). On benchmarking functions for genetic algorithms. International journal of computer mathematics, 77(4), 481-506.

[47] Van den Bergh, F., \& Engelbrecht, A. P. (2006). A study of particle swarm optimization particle trajectories, Information sciences, 176(8), 937-971. 